\title{
Noise Induced Hearing Loss in Indian Railway Travelling Ticket Examiners
}

\section{Athira Rajan', Srikanth Nayak ${ }^{2,3}$, Lidiya Mathew ${ }^{4}$, Rahana Shiju $^{4}$ and Sathish Kumar ${ }^{5 *}$}

${ }^{1}$ Assistant Professor, Department of Audiology and Speech-Language Pathology,

Nitte Institute of Speech and Hearing, Mangalore, India

${ }^{2}$ Assistant professor, Department of Audiology and Speech-Language Pathology,

Yenepoya University, Mangalore, India

${ }^{3}$ Lecturer, Department of Audiology and Speech-Language Pathology, Nitte

Institute of Speech and Hearing, Mangalore, India

${ }^{4}$ Student Intern, Department of Audiology and Speech-Language Pathology, Nitte

Institute of Speech and Hearing, Mangalore, India

${ }^{5}$ Lecturer, Department of Audiology and Speech-Language Pathology, Madras ENT

Research Foundation, Chennai, India

*Corresponding Author: Sathish Kumar, Lecturer, Department of Audiology and Speech-Language Pathology, Madras ENT Research Foundation, Chennai, India.
Received: July 26, 2021

Published: August 16, 2021

(C) All rights are reserved by Sathish Kumar., et al.

\section{Abstract}

Background: Railway Travelling Ticket Examiner (TTE) is an employee in Indian railways who examines 'passenger's tickets during the journey in trains. TTEs are more susceptible to noise-induced hearing loss as they work for around 8 hours per day on the train and have continuous exposure to loud noise during their working hours. So, the present study aimed to assess the hearing sensitivity of the Indian Travelling Ticket Examiner.

Materials and Methods: The study included thirteen railway TTEs for hearing assessment. The hearing sensitivity was assessed by obtaining a detailed case history, otoscopic examination, pure tone audiometry and tympanometry.

Results: The mean pure tone average was $22.5 \pm 6.03 \mathrm{dBHL}$ and $19.42 \pm 7.06 \mathrm{dBHL}$ in the right and left ear, respectively. All the subjects had minimal to mild sensorineural hearing loss in both ears. The degree of hearing loss increased at high frequencies $(4$ and 8 $\mathrm{kHz})$.

Conclusion: The affected hearing thresholds in TTEs depicts a significant correlation between their service experience and hearing loss. The positive correction between TTEs work experience and hearing loss may lead to a handicapping situation in their professional and personal life. Hence the enrollment of railway TTEs in an appropriate and effective Hearing Conservation Program is a necessity.

Keywords: Railway Travelling Ticket Examiner; Noise Exposure; Noise-Induced Hearing Loss; Puretone Audiometry; Hearing Conservation

\section{Abbreviations}

TTE: Travelling Ticket Examiner; NIHL: Noise-Induced Hearing Loss; PTA: Pure Tone Audiometry

\section{Introduction}

Noise is an unpleasant sensation formed by several sound waves with anarchically distributed amplitude and phase ratios 
[1]. The spread of rapid industrialization and urbanization across the world resulted in employing more and more individuals in various work sectors to boost socio-economic growth, which led to a rise in the prevalence of occupational hearing loss. Occupational noise-induced hearing loss can be defined as a partial or complete hearing loss in one or both ears due to one's employment [2]. The degree of hearing loss depends on the characteristics of exposure in the level, time and frequency domains, as well as the individual's susceptibility. Occupational noise accounts for $16 \%$ of hearing loss in adults, ranging from $7 \%$ to $21 \%$ [3]. Many workers including those engaged in industrial works and drivers of heavy vehicles, traffic police officers, aircrew and railroad service employees including locomotive crew, are occupationally exposed to noise and are at potential risk of developing occupational NIHL $[2,4]$.

Excessive noise exposure leads to hearing loss which in turn affects speech comprehension, resulting in personal disabilities, handicaps and behavioural changes [1]. Excessive noise exposure leads to various adverse effects on an individual's health, which are grossly classified into auditory and non-auditory effects. The auditory effect of continuous and excessive noise exposure causes permanent damage to the cochlea, especially the basal turn of the cochlea, which can lead to noise-induced hearing loss (NIHL) [5]. According to Nandi and Dhatrak [2], "NIHL generally denotes the cumulative, permanent loss of hearing that develops gradually after months or years of exposure to high levels of noise". Other auditory effects include tinnitus, diplacusis, and hyperacusis. Non-auditory effects include physiological effects such as increased blood pressure, variation in heart rate, vasoconstriction and disturbances in sleep patterns, performance and mental health of an individual [5].

Indian Railway is the largest employer among the nation's public sector, with around 1.3 million employees [6]. The noise measurements in the railway companies revealed an employee is exposed to an average 8 hours noise exposure level of $70-85 \mathrm{dBA}$ with peak exposures reaching $130-140 \mathrm{dBC}$ [7]. A study conducted in one of the railway station platforms in India reported excessive sound pressure level (SPL) exceeding the American Conference of Government Industrial Hygienists (ACGIH) standards.

Considering these higher levels of noise, it is evident that railway workers are at risk of developing permanent hearing loss. Travelling Ticket Examiner (TTE) is an employee in Indian railways who examines passenger's tickets during train journeys. TTEs work for 8 hours/shift and are exposed to excessive noise and develop NIHL. Hence, there is a strong need to rule out the occupational noise-induced hearing loss in Railway TTEs. Further, it is essential to identify the relationship between their occupation and hearing status and establish appropriate preventive measures if required.

\section{Aim of the Study}

Hence, the current study aims to assess the NIHL in Indian Travelling Ticket Examiners.

\section{Methods}

\section{Participant selection}

Thirteen active male TTEs between 35 to 50 years with a minimum work experience of five years were recruited for this study. The otoscopic and tympanometry examination was performed to rule out conductive pathology. The individuals with a history of middle ear disorders or any systemic illness like diabetes mellitus and hypertension were excluded. The study protocol was approved by the Institutional ethical committee of Nitte Institute of Speech and Hearing, Mangalore.

\section{Procedure}

The TTEs were priorly informed regarding the study, and consent was obtained. The study was conducted in a noise-controlled room near the railway station. Prior to the pure tone audiometry, a detailed case history, otoscopic examination and immittance audiometry was performed to rule out conductive pathologies by an experienced audiologist. The TTEs who met the inclusion criteria were subjected to pure tone audiometry. Calibrated Alps Audiometer AD2100 with TDH 39 headphone and B-71 bone vibrator for the hearing threshold estimation. Using a modified Hughson-Westlake procedure, the hearing sensitivity of TTEs were measured by a single experienced audiologist in a noise-controlled room. All the subjects were advised to rest for 8 hours post-work to obtain the best thresholds across the hearing frequency range (500 to 8000 $\mathrm{Hz}$ for air conduction and 500 to $4000 \mathrm{~Hz}$ for bone conduction). The pure tone average thresholds $(0.5,1,2$ and $4 \mathrm{k} \mathrm{Hz})$ were used to describe the hearing sensitivity. The bone conduction thresholds measured to evaluate the presence of conductive hearing loss were not included for analysis. 
TTEs are considered to have normal hearing during the time of recruitment based on the Indian railway recruitment policy [8]. Hence, all the TTEs were considered to have a pure tone average of $15 \mathrm{~dB}$ HL (theoretical normal hearing threshold) during the time of recruitment and hearing thresholds obtained in this study were compared with theoretical normal hearing thresholds using a onesample t-test. The correlation between work experience and the hearing threshold was analyzed using Pearson's correlation. All the statistical tests were performed using SPSS v17.0.

\section{Results}

Thirteen subjects between the age range of 35 to 50 years with a mean age of $46.08 \pm 5.34$ years participated in this study. The work experience of the TTEs ranged from 9 years to 30 years, with an average of $19.08 \pm 8.7$ years. The descriptive statistics for pure tone thresholds in both ears are mentioned in table 1 . The mean pure tone average in TTEs was $22.5 \pm 6.03 \mathrm{dBHL}$ in the right ear and $19.42 \pm 7.06 \mathrm{dBHL}$ in the left ear. One sample t-test showed a significant increase in the hearing threshold levels for 1, 2, 4 and $8 \mathrm{kHz}$ in the right ear and 4 and $8 \mathrm{kHz}$ in the left ear compared to the theoretical normal hearing threshold. The degree of hearing loss was higher at 4 and $8 \mathrm{kHz}$ than at lower frequencies. The Pearson's correlation revealed a significant association between service experience and hearing threshold ( $r=0.24, \mathrm{p}<0.05)$. Out of the thirteen TTEs, five TTEs reported tinnitus as a complaint during the case history.

\begin{tabular}{|c|c|c|}
\hline $\begin{array}{c}\text { Frequency } \\
\text { (Hz) }\end{array}$ & $\begin{array}{c}\text { Right ear } \\
\text { thresholds } \\
\text { (dBHL) }\end{array}$ & $\begin{array}{c}\text { Left ear thresholds } \\
\text { (dBHL) }\end{array}$ \\
\hline 500 & $17.3 \pm 4.2$ & $16.15 \pm 4.16$ \\
\hline 1000 & $19.6 \pm 6.2^{*}$ & $14.61 \pm 7.48$ \\
\hline 2000 & $20 \pm 6.77^{*}$ & $17.69 \pm 7.53$ \\
\hline 4000 & $33.84 \pm 11.92^{*}$ & $29.61 \pm 15.06^{*}$ \\
\hline 8000 & $30.77 \pm 17.54^{*}$ & $31.15 \pm 17.46^{*}$ \\
\hline
\end{tabular}

Table 1: Mean and standard deviation of pure tone thresholds of right and left ears.

*: Denotes $\mathrm{p}<0.05$ for one sample t-test.

\section{Discussion}

The present study explored Occupational Noise-Induced Hearing Loss in Indian Railway Travelling Ticket Examiners. Thirty thousand five hundred thirty-five ticket examiners were employed in the Indian Railways according to the data of 2017-18 [9]. Although TTEs are at higher risk of developing noise-induced hearing loss due to their continuous and excessive exposure to noise, the literature support for this is limited. Few studies available in the literature analyzed the hearing sensitivity among loco pilots, and very limited information is available on NIHL in TTEs.

The present study results showed minimal to mild sensorineural hearing loss in TTEs and were found to have a strong correlation with their work experience. The present study findings substantiate the findings in the literature reporting NIHL leads to sensorineural hearing loss. Similar findings are reported in loco pilots of the railway sector $[10,11]$. Apart from this, a survey conducted on Iranian train drivers revealed $15.8 \%$ having a high frequency and $3.3 \%$ having low frequency hearing loss [12]. Similarly, poor hearing thresholds in Taiwan train drivers were reported when compared with the general Taiwanese population [13]. All these findings confirm that people working in the railway sector have a higher risk of developing NIHL.

The subjects of the present study had hearing loss increasing significantly in both ears above $4 \mathrm{kHz}$. McBride and Williams stated, "possible explanation for the existence of hearing loss above $2 \mathrm{kHz}$ would be the fact that NIHL preferentially affects the high frequencies, with the hearing loss beginning at around $4 \mathrm{kHz}$ before spreading into the lower frequencies" [14]. The present study also depicts an association between the degree of hearing loss and the duration of noise exposure. The TTEs with higher losses were those with more work experience in the railways. This is in accordance with the study conducted on loco pilots in India, which revealed a mild to profound sensorineural hearing loss in the loco pilots were directly proportional to their service tenure [15].

In the present study, out of the thirteen TTEs, five TTEs reported tinnitus during the case history, supporting the reports in the literature that tinnitus is observed in individuals after acute or chronic noise exposure [16]. The findings from the present study contradict the findings of Lie., et al. who observed normal hearing in Norwegian train drivers [7]. A possible explanation for this discrepancy could be the improved engineering and maintenance of types of machinery and the more relaxed schedule of the 'employees' working hours in the Norway railway system when compared to the Indian scenario. The study conducted by Clark and Popelka 
also found no significant difference between the hearing sensitivity of railroad train crew members and that of the subjects employed in the control group [17].

Further, upon exploration in our study, it was found that there is a lack of awareness regarding occupational hazards among TTEs. OSHA's mandated Hearing Conservation Amendment stated that the employers must provide Hearing Protection Devices (HPDs) to the employees exposed to the noise level of $85 \mathrm{~dB}$ or above over 8 hours [18]. HPDs can attenuate the level of noise, reaching the employees' ears when worn appropriately. However, the subjects employed in the present study were not using any kinds of HPDs during their work hours. Implementing adequate engineering and administrative controls, along with the use of appropriate HPDs, can lower the risk of NIHL in the railway employees. Large-scale studies need to be conducted to understand the effect of noise exposure in Indian railway employees, which can help develop hearing conservation protocols. Hence, the present study throws light on creating awareness among the TTEs regarding the usage of ear protective devices and also on the need for the annual audiological evaluation to monitor the hearing status among the TTEs.

\section{Conclusion}

The present study aimed to investigate the relationship between the hearing status of the TTEs and their occupation. The results of the study point to the fact that excessive and continuous noise exposure of the Indian railway TTEs makes them vulnerable to develop hearing loss. This is in accordance with previous study results that reported hearing loss in the Indian Loco pilots [10]. The interactive effects of occupational NIHL, ageing and recreational noise exposure may ultimately result in a handicapping situation in these railway employees' professional and personal lives. Hence the enrollment of Railway employees in an appropriate and effective Hearing Conservation Program is a necessity.

\section{Acknowledgement}

We thank the Department of Audiology and Speech-Language Pathology, Nitte Institute of Speech and Hearing, Mangalore, for permitting us to conduct the study.

\section{Conflict of Interest}

The authors declare no potential conflict of interest.

\section{Funding Support}

No funding sources.

\section{Bibliography}

1. De Almeida SI., et al. "Natural history of occupational hearing loss induced by noise". Revista da Associacao Medica Brasileira 46.2 (1992): 143-158.

2. Nandi SS and Dhatrak SV. "Occupational noise-induced hearing loss in India”. Indian Journal of Occupational and Environmental Medicine 12.2 (2008): 53.

3. Nelson DI., et al. "The global burden of occupational noise-induced hearing loss". American Journal of Industrial Medicine 48.6 (2005): 446-458.

4. Azizi MH. "Occupational noise-induced hearing loss". Journal of Occupational and Environmental Medicine (2010).

5. Basner M., et al. "Auditory and non-auditory effects of noise on health". The Lancet 383.9925 (2014): 1325-1332.

6. Indian railways. Indian railways year book (2018-19) (2019).

7. Lie A., et al. "Hearing status among Norwegian train drivers and train conductors". Occupational Medicine 63.8 (2013): 544-548.

8. Indian railways. Medical examination in Indian railways (1973).

9. Economic times. How railways plan to curb ticketless travellers. The Economic Times (2019).

10. Saxena A. "Noise Induced Hearing Loss in Indian Railway Loco Pilots: Are We Aware?" Open Journal of Otolaryngology 4.2 (2018):18-21.

11. Loginova V. "Hygienic assessment of working conditions and occupational risk for workers health at railway transport objects". Health 2 (2017): 89-93.

12. Loukzadeh Z., et al. "Fitness-for-work assessment of train drivers of Yazd railway, central Iran". Journal of Occupational and Environmental Medicine 4.3 (2013): 199-157.

13. Wang K., et al. "The Study on the Environmental Noise Exposure and Hearing Loss of Train Drivers in Taiwan". Epidemiology 18.5 (2007): S56. 
14. McBride DI and Williams S. "Audiometric notch as a sign of noise induced hearing loss". Occupational and Environmental Medicine 58.1 (2001): 46-51.

15. Waghmare S., et al. "Evaluation of noise induced hearing loss in rail engine drivers using BERA". European Journal of Biomedical and Pharmaceutical Sciences 2.4 (2015): 502-510.

16. Alberti P. "Tinnitus in occupational hearing loss: nosological aspects". Journal of Otolaryngology 1.16 (1987): 34-35.

17. Clark WW and Popelka GR. "Hearing levels of railroad trainmen”. Laryngoscope 99.11 (1989): 1151-1157.

18. OSHA. Occupational noise exposure: Hearing conservation amendment". Federal Register (1981): 4078-4085.

Volume 3 Issue 9 September 2021

(c) All rights are reserved by Sathish Kumar., et al. 